\title{
Application of SPOD analysis to PIV data obtained in the wake of a circular cylinder undergoing vortex induced vibrations
}

\author{
Christopher M. O’Neill ${ }^{1}$, Yannick Schubert ${ }^{2}$, Moritz Sieber ${ }^{2}$, Robert \\ Martinuzzi $^{1}$, Chris Morton ${ }^{1 *}$ \\ ${ }^{1}$ Department of Mechanical Engineering, University of Calgary, Calgary, Canada \\ ${ }^{2}$ Institut für Strömungsmechanik und Technische Akustik, Hermann-Föttinger-Institut, Technische \\ Universität Berlin, Berlin, Germany \\ * chris.morton@ucalgary.ca
}

\begin{abstract}
Vortex induced vibrations (VIV) of a circular cylinder have been investigated experimentally using a cyberphysical apparatus with $m^{*}=8, \zeta=0.005$, and $R e=4000$. This study considers the application of proper orthogonal decomposition (POD) and spectral POD (SPOD) analysis to the wake dynamics of the low-massratio VIV of a circular cylinder in the lower branch at $U^{*}=7.5$. SPOD has been previously shown to better separate frequency-centered modal dynamics, compared to POD. Coherent POD and SPOD modes were compared and the newly separated third SPOD mode pair was found to have a periodicity characteristic of vortex shedding and a peak in the temporal coefficient spectra at $S t=f D / U_{\infty}=0.2248$. The literature has identified that the wake dynamics within the lower branch are synchronized to the cylinder motion; however the present study suggests that some hidden dynamics persist at the Strouhal frequency. Low order models based on the first eight POD and SPOD modes were compared, and it was found that the filtering operation in SPOD removes the uncorrelated stochastic energy component of the POD modes while producing a comparable representation of the coherent deterministic part of the wake dynamics. Using SPOD to separate the distinct frequency-centered dynamics into unique, interpretable mode pairs will simplify future efforts to develop sparse dynamical models of the flow.
\end{abstract}

\section{Introduction}

Vortex-induced vibration of a circular cylinder is a fluid-structure interaction problem presenting engineering challenges to the design of civil structures (e.g., cable-tensioned bridges) as well as mechanical engineering devices (e.g., heat exchangers). Comprehensive reviews of VIV can be found in the works of Sarpkaya (2004) and Williamson and Govardhan (2004). An elastically mounted cylinder in cross-flow is susceptible to vortex induced vibrations when the vortex shedding frequency is near the natural frequency of the system. The resonance of the structure and the associated 'lock-in' phenomenon occur when the vortex shedding frequency is synchronized to the vortex induced oscillations of the body. The structural dynamics of the cylinder motion are given by:

$$
m \ddot{y}+c \dot{y}+k y=F_{y}
$$

where $m, c, k$ are the structural mass, damping, and spring constant respectively. $F_{y}$ is the transverse component of the fluid force on the cylinder. The natural frequency of the system is defined as $f_{N}=\sqrt{k / m}$. The following section discusses the contributions of the cylinder wake dynamics to the amplitude and frequency response of the system, and approaches in modeling of this fluid-structure interaction.

\subsection{Cylinder VIV Wake Modes, Amplitude and Frequency Response}

The VIV amplitude response $\left(A^{*}=A / D\right.$, where $D$ is the cylinder diameter) for a low mass-damping system $\left(m^{*} \zeta<0.1, m^{*}=4 m / \rho \pi D^{2} L, \zeta=c / 2 \sqrt{\mathrm{km}}\right)$ across a range of reduced velocities $\left(U^{*}=U_{\infty} / f_{N} D\right)$ is 
characterized by an initial branch, upper branch, lower branch, and a desynchronization region (Khalak and Williamson, 1997). The topology of the wake for a given reduced velocity and response amplitude has been characterized in controlled motion experiments and was classified into vortex shedding modes using phaseaveraging as described by Morse and Williamson (2009). The transitions between branches are somewhat sensitive to $m^{*} \zeta$; for consistency with the literature, the following description of the amplitude response follows Khalak and Williamson (1997). A description of the amplitude response associated with the $m^{*} \zeta$ of the apparatus used in this study can be found in Riches and Morton (2018).

In the initial branch $\left(2 \lesssim U^{*} \lesssim 4.75\right)$, with increasing $U^{*}$ the frequency of vortex shedding approaches the natural frequency of the system causing an increase in the oscillation amplitude. The vortex shedding frequency and the natural frequency are both present within the cylinder position spectrum. Following the nomenclature of Morse and Williamson (2009), this branch is characterized by 2S-type vortex shedding in which single counter-rotating vortices are shed alternately from the cylinder sides.

When the reduced velocity is increased further above $U^{*} \approx 4.75$, the amplitude response of the cylinder transitions to the upper branch, where the vortex shedding frequency becomes synchronized with the natural frequency of the system and structural resonance occurs leading to large oscillations. The wake topology in the upper branch is characterized by $2 P_{O}$-type vortex shedding, which consists of pairs of counter-rotating vortices. These pairs are shed alternately from the cylinder sides with the trailing vortex being significantly weaker than the leading vortex (Morse and Williamson, 2009).

The lower branch occurs for $6 \lesssim U^{*} \lesssim 9.5$, and is characterized by a slightly-lower steady state amplitude response compared to the upper branch. The wake topology in the lower branch is characterized by $2 \mathrm{P}-$ type vortex shedding, where pairs of equally strong counter-rotating vortices shed from alternating sides of the cylinder. While oscillation and shedding remain synchronized, the peak in the cylinder oscillation spectra shifts to be slightly higher than the natural frequency. This synchronization of the natural and oscillation frequencies is known as 'lock-in'. According to Sarpkaya(2004), lock-in occurs when the motion of the cylinder becomes a dominant factor in the interaction of the separated shear layers that causes vortex shedding in the wake. The focus of this paper will be further analysis of the wake topology at $U^{*}=7.5$ in the lower branch.

For reduced velocities above $U^{*} \approx 9.5$, the vortex shedding frequency is no longer synchronized with the natural frequency, and the amplitude response of the system decays. In the cylinder position spectra, the vortex shedding frequency and the natural frequency re-emerge as separate frequencies.

\subsection{Further analysis of Cylinder VIV and Low Order Modeling}

Beyond the phase-average based characterization of the topology of the wake by Morse and Williamson (2009), VIV has been studied using several techniques including Proper Orthogonal Decomposition (POD) Riches et al. (2018), Dynamic Mode Decomposition (DMD) Freire et al. (2015), and deep learning Raissi et al. (2019), based on time-resolved Particle Image Velocimetry (PIV) data. In addition to characterizing the flow topology, there is an interest in using these methods to develop physics-based models of the flow state. POD has been used to develop low order models (LOM) of the flow state, such as described in Liberge and Hamdouni (2010) where a 6-mode LOM was found to accurately reconstruct the velocity field for a computational study of cylinder VIV at $R e=1690$. Riches et al. (2018) applied POD to cylinder VIV in the initial $(R e=3100)$ and upper $(R e=4100)$ branches and found that 6 - and 7-mode LOMs, respectively, represented the coherent part of the cylinder wake. Riches et al. (2018) also investigated the analytical relationships between the POD modes in order to develop a model of the flow. As explored in Riches et al. (2018), discerning which modes correspond to the coherent dynamics can be challenging. For example, a temporal Gaussian filter was applied to data in a pre-processing step in order to separate low-frequency and high frequency dynamics which could not be identified using POD alone. Spectral Proper Orthogonal Decomposition (SPOD)(Sieber et al. 2016) involves a more robust filtering process, and has shown promise in decoupling modal dynamics for bluff body wakes. SPOD recently was applied to the wake of a FX63137 airfoil with a Gurney flap, at $R e_{c}=180 \times 10^{3}$, and was found to produce a simpler wake model by decoupling the interaction of the upstream vortex from the primary vortex shedding (Sieber et al., 2016).

\subsection{Objective}

The objective of this paper is to consider the application of SPOD to decomposition of the lower branch of VIV, and illustrate the advantages SPOD compared to POD in extracting dynamically relevant modes. This paper will first examine the effect of the SPOD filter length on the high energy modes, followed by 
analyzing the cylinder wake. POD-based and SPOD-based LOM will be constructed and compared to examine the benefits SPOD offers in terms of representing the flow state.

\section{Methods}

Experiments were carried out in a water tunnel facility at the University of Calgary. A cyber-physical apparatus was employed to study VIV of a circular cylinder, as described in Riches and Morton (2018). Following the works of Hover et al. (1997), Mackowski and Williamson (2011), and Derakhshandeh et al. (2015), the apparatus operates by real-time simulation of the differential equation of the cylinder's motion, given in Equation 1. The transverse component of the force on the cylinder $\left(F_{y}\right)$ is taken as the input, and the structural mass, damping, and spring constant are applied in software to compute the desired kinematics. The cylinder is then forced to follow the desired kinematics using a motorized traverse. The transverse force on the cylinder was measured using an ATI Mini40 force transducer with a resolution of $0.005[N]$. The traverse was driven using a ClearPath digital brushless servo motor (MCVC-2341P) and the position was measured using an Omron optical encoder with a position resolution of $0.0375[\mathrm{~mm}]$. The block diagram of Equation 1 was used to compute the kinematics, and is shown below in Figure $1 \mathrm{p}$.
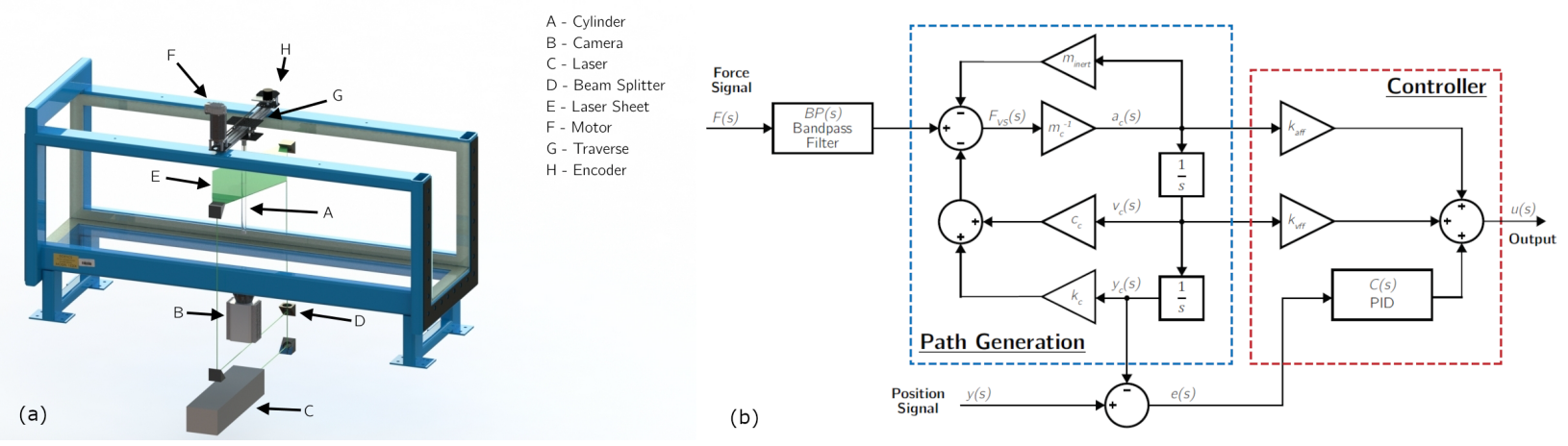

Figure 1: (a) Experimental apparatus placed in a section of the University of Calgary water channel, with PIV imaging setup shown. (b) Cyber-physical apparatus path generation algorithm and system controller. Additional details on this system can be found in Riches (2018). Figure 1 is adapted from Riches and Morton (2018).

The parameters investigated in this study correspond to $m^{*}=8, \zeta=0.005, R e=4000$, and $U^{*}=7.5$. The free-stream velocity was set to fix the $R e$, while $U^{*}$ was set by altering the natural frequency of the system. The structural natural frequency of the system is defined in air. The natural frequency and damping ratio were confirmed based on the system accurately reproducing an oscillating free decay after release from an initial displacement, as shown in Riches and Morton (2018). Good agreement was found between the amplitude and frequency response of the cyber-physical apparatus with data from Govardhan and Williamson (2006) and Klamo et al. (2006), as discussed in Riches and Morton (2018).

Time-resolved two-component planar PIV measurements were obtained for a plane located at the midspan of the cylinder, as shown in Figure 17. Images were captured using a high speed Phantom Miro M340 digital camera focused on the mid-span plane illuminated by a laser sheet generated by a Photonics $20 \mathrm{~mJ}$ $\mathrm{Nd}$ :YLF high repetition rate pulsed laser with a wavelength of $l=527[\mathrm{~nm}]$. PIV images were processed using LaVision DaVis 8.3 software. A total of $N=4767$ vector fields were obtained at a sample frequency of $24 \mathrm{~Hz}$, spanning approximately 290 oscillation periods of the cylinder. Velocity vector uncertainties were calculated using the correlation statistics method of Wieneke (2015), as employed within DaVis 8.3 and were an average of $2.2 \%$ in the free stream and $4.0 \%$ in the wake.

\subsection{Proper orthogonal decomposition}

The velocity field $u(\mathbf{x}, t)$ is split into mean $\bar{u}(\mathbf{x})$ and fluctuating $u^{\prime}(\mathbf{x}, t)$ components and then fluctuating component is decomposed into a finite sum of spatial eigenfunctions $\phi_{i}(x)$ multiplied by a time-dependent 
temporal modal coefficients $a_{i}(t)$ following the snapshot POD method of Sirovich (1987):

$$
u(\mathbf{x}, t)=\bar{u}(\mathbf{x})+u^{\prime}(\mathbf{x}, t)=\bar{u}(\mathbf{x})+\sum_{i=1}^{N} \phi_{i}(\mathbf{x}) a_{i}(t)
$$

To compute the correlation between snapshots, the $L^{2}$ inner product is first defined using $\langle$,$\rangle . Applying the$ inner product to some arbitrary velocity fields $p(\mathbf{x})$ and $q(\mathbf{x})$ gives:

$$
\langle p(\mathbf{x}), q(\mathbf{x})\rangle=\int_{\Omega} p(\mathbf{x}) q(\mathbf{x}) d \Omega
$$

where $\Omega$ defines the spatial volume where the correlation is integrated. If velocity fields $p(\mathbf{x})$ and $q(\mathbf{x})$ are orthonormal, then $\langle p(\mathbf{x}), q(\mathbf{x})\rangle=\delta_{i, j}$. Using the inner product, the elements of the snapshot correlation matrix $R_{i, j}$ can be computed according to:

$$
R_{i, j}=\frac{1}{N}\left\langle u^{\prime}\left(\mathbf{x}, t_{i}\right), u^{\prime}\left(\mathbf{x}, t_{j}\right)\right\rangle
$$

The temporal coefficients $a_{i}(t)$ and mode energies $\lambda_{i}$ are determined from the eigenvectors and eigenvalues of the correlation matrix:

$$
R a_{i}(t)=\lambda_{i} a_{i}(t)
$$

The spatial modes $\phi_{i}(\mathbf{x})$ are computed from the projection of the snapshots onto the temporal coefficients:

$$
\phi_{i}(\mathbf{x})=\frac{1}{N \lambda_{i}} \sum_{j=1}^{N} a_{i}\left(t_{j}\right) u^{\prime}\left(\mathbf{x}, t_{j}\right)
$$

The spatial modes are orthonormal by construction and therefore they satisfy the condition:

$$
\left\langle\phi_{i}(\mathbf{x}), \phi_{j}(\mathbf{x})\right\rangle=\delta_{i, j}
$$

The process of decomposing the fluctuating field using POD produces orthogonal modes and coefficients such that a minimal number of modes contain the maximum amount of energy within the field; however, the modes may contain energy at multiple frequencies and therefore may not represent a specific dynamical structure in the wake. Once a decomposition of the flow has been performed, a low-order model of the field $u_{L O M}^{\prime}(\mathbf{x}, t)$ can be created using an expansion of the first $N_{t}$ POD modes associated with coherent dynamics in Equation 2, thus yielding the coherent part of the fluctuating velocity field (Rowley (2005); Noack et al. (2011); Bourgeois et al. (2013)). This low order model can be found according to:

$$
u_{L O M}^{\prime}(\mathbf{x}, t)=\sum_{k=1}^{N_{t}} \phi_{i}(\mathbf{x}) a_{i}(t)
$$

\subsection{Spectral Proper Orthogonal Decomposition}

Spectral POD, as described in Sieber et al. (2016), is an alternative decomposition method to POD. Beginning with the snapshot correlation matrix defined in Equation 4, a low-pass filtering is performed along the diagonal to form a filtered correlation matrix, which has elements $S_{i, j}$ given by:

$$
S_{i, j}=\sum_{k=-N_{f}}^{N_{f}} g_{k} R_{i+k, j+k}
$$

where $N_{f}$ is the filter length, and $g_{k}$ is an entry of the 1-D filter kernel $g$ of length $2 N_{f}+1$. Sieber et al. (2016) showed that in the case of a box-filter with equal coefficients given by $g_{k}=1 /\left(2 N_{f}+1\right)$, that with $N_{f}=0$, SPOD is equivalent to POD. Further with $N_{f}=N$, SPOD is equivalent to the discrete fourier transform (DFT). After the filtering, remaining steps in the decomposition proceed as described above in POD, yielding a set of spatial modes and temporal coefficients. Based on Sieber et al. (2016), a Gaussian filter kernel is used in the following results, due to preferable properties of smooth roll off, except for $N_{f}=0$ and $N_{f}=4767$, where a box filter was used to recover POD and the DFT. Mode pairing was performed as described in Sieber et al. (2016), including identification of the primary frequency for the temporal coefficient of each mode pair. 


\section{Results}

\subsection{SPOD Filter Length}

Investigation of the dynamical features of the flow was first carried out using a POD-based approach similar to that of Riches et al. (2018). This corresponds to a filter length of $N_{f}=0$ in Equation 9. The choice of filter length $0<\bar{N}_{f}<N$ represents the trade off between the spectrally agnostic POD and spectrally pure DFT. In a practical sense, as the filter length is increased from zero (POD), the spectral bandwidth of the mode temporal coefficients is constrained. The resulting spatial modes are affected through the projection operation, and energy outside the mode spectral bandwidth is redistributed to other modes.

The filter length parameter $N_{f}$ determines the number of 2D PIV vector fields averaged by the filter and thus proper selection depends on both the sample rate and the frequency of the underlying dynamics. The filter length can be examined with reference to the number of 2D PIV vector fields for one oscillation of the cylinder $\left(N_{\text {osc }}\right)$. The cylinder oscillation frequency $f_{\text {osc }}=1.753[\mathrm{~Hz}]\left(S t=f_{\text {osc }} D / U_{\infty}=0.15\right)$ was determined based on spectral analysis of the cylinder position signal, see Figure 2. The number of vector fields capturing a given oscillation cycle is:

$$
N_{\text {osc }}=\frac{f_{s}}{f_{\text {osc }}}=\frac{24}{1.753}=13.69 \approx 14
$$

The effects of the filter length on the spatial modes and temporal coefficient spectra was examined over a wide range of $N_{f}$. For brevity only $N_{f}=\left[0,4 N_{o s c}, 5 N_{o s c}, N\right]$ are shown in Figure 3. Figure 3(i) shows the energy distribution of mode pairs (y-axis) and their associated frequencies (x-axis). The size and color of the markers represents the correlation between the modes in the mode pair; pairs with large yellow markers are well correlated. Figure 3 (ii,iv,vi,viii) shows the u-component of the first four most energetic POD spatial mode pairs, or the equivalent SPOD mode pair. Figure $3($ iii,v,vii,ix) shows the temporal coefficient PSDF corresponding to the mode pairs in Figure 3 (ii,iv,vi,viii).

The spatial mode in Figure 3 a(ii) shows a periodicity that is characteristic of vortex shedding, and the corresponding temporal coefficient spectrum for the pair shown in Figure 3a(iii) has a peak at the $S t=$ $f D / U_{\infty}=0.15$, which corresponds to the oscillation frequency of the cylinder. Additional energy content in this pair is spread across multiple frequencies, including a secondary peak at $f D / U_{\infty} \approx 0.2$. The structures in the spatial mode in Figure 3 (iv) show half the spatial wavelength compared to Figure 3 (ii) and the PSDF of the temporal coefficient in Figure $3 \mathrm{a}(\mathrm{v})$ shows a peak at $S t=f D / U_{\infty}=0.30$; therefore, this mode pair corresponds to the second harmonic of the vortex shedding at the oscillation frequency. The second harmonic mode pair has energy content spread across multiple frequencies, including a secondary peak at $f D / U_{\infty} \approx$ 0.2. The spatial modes in Figure $3 \mathrm{a}$ (vi) and (viii) contain large scale features and the corresponding temporal coefficient spectrum contains a broad range of scales, including broad peaks at $f D / U_{\infty} \approx 0.1$ and $f D / U_{\infty} \approx$ 0.2 .

As the filter length is increased in the range $0 \leq N_{f} \leq 4767$, the trend towards decreasing spectral bandwidth can be observed by comparing the temporal coefficient spectra in Figures 3(iii,v,vii,ix). For example in Figure $3 \mathrm{~b}(\mathrm{v})$, most of the mode energy in the temporal coefficient spectra is contained in the range approximately $0.26 \leq f D / U_{\infty} \leq 0.35$. Increasing the filter length from $N_{f}=56$ to $N_{f}=70$, in Figure $3 \mathrm{k}(\mathrm{v})$ most of the energy is now contained within $0.28 \leq f D / U_{\infty} \leq 0.33$. Thus as the filter length increases, the temporal coefficients for the first two mode pairs are constrained to progressively narrower frequency bands without significant energy loss as shown by comparison of Figures 3(i).

Within the temporal coefficient spectra, the secondary peak observed in Figure $3 \mathrm{a}(\mathrm{v})$ and $3 \mathrm{a}(\mathrm{vi})$ at $f D / U_{\infty} \approx 0.2$ is absent for $N_{f}>56$. The broad peak in Figure $3 \mathrm{a}$ (ix) is significantly weakened for $N_{f}=56$ and almost absent for $N_{f}=70$, suggesting $N_{f} \geq 70$ is required to separate the third mode pair from the fourth mode pair. Comparing Figure $3 \mathrm{~A}-\mathrm{c}(\mathrm{vii})$, the peak at $f D / U_{\infty} \approx 0.2$ is well defined for $N_{f} \geq 56$. Increasing the filter length above $N_{f}=70$ further constrained the frequency content of the third mode pair and progressively reduced the energy of the mode pair. The frequency bandwidth of the third mode pair is broader than the first two mode pairs, thus further constraining the bandwidth of this mode would only exclude relevant dynamics and their energy content. Higher filter lengths such as $N_{f}=112$ offered no clear benefit in separating the dynamics associated with the fourth mode pair. Thus $N_{f}=70$ was chosen based on the trade off between increasing energy losses of the third mode pair and frequency separation of the fourth mode pair. 


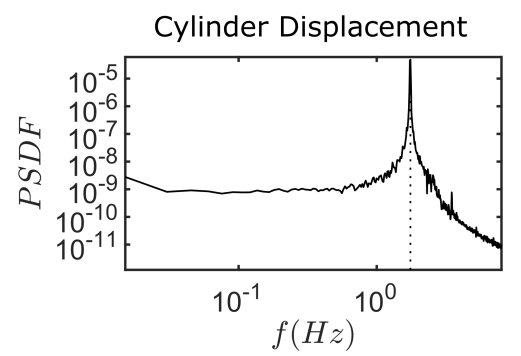

Figure 2: Power Spectral Density Function (PSDF) for the cylinder displacement signal. The dotted line is located at $f=1.753 \mathrm{~Hz}$, and corresponds to the cylinder oscillation frequency $\left(f_{\text {osc }}\right)$.

\subsection{Analysis of Wake Dynamics for SPOD with $N_{f}=70$}

Following selection of an appropriate filter length $\left(N_{f}=70\right)$ for separating the relevant dynamics, it is important to consider what new fundamental physical insights have been established. For this purpose, Figure 4 shows a direct comparison of the spatial modes and temporal coefficient spectra between POD (a) and SPOD (b). From left to right, each row of Figure 4 consists of the u- and v-component of the spatial modes for the first and second modes in the pair, followed by the temporal coefficient spectra. As previously discussed, the mode pairs $(1,2)$ and $(3,4)$ in both POD and SPOD have spatial modes and temporal coefficient spectra characteristic of the oscillation frequency and its second harmonic, respectively. The POD mode pairs are contaminated with low and high frequency content, with multiple peaks visible in the spectra for all four mode pairs in Figure 4a. Moreover, the third and fourth mode pairs $(5,6$ and 7,8) are difficult to interpret and contains a mixture of low frequency content with broad local maxima located at $f D / U_{\infty} \approx 0.1$ and $f D / U_{\infty} \approx 0.2$. In contrast, the SPOD mode pair $(5,6)$ has a spatial wavelength and mode shape typical of a vortex shedding phenomena, and a well defined peak in the frequency domain at $S t=f D / U_{\infty}=0.2248$, which is close to the expected Strouhal number of $\sim 0.21$ for a stationary cylinder at $R e=4000$ (Norberg, 2003). These results suggest that SPOD modes $(5,6)$ represent a natural vortex shedding process which is not synchronized to the cylinder oscillation, and that this process may persist within the lower branch. The SPOD mode pair $(7,8)$ has a longer spatial wavelength with a peak in the frequency domain at $f D / U_{\infty}=$ 0.0753 , which corresponds closely to the beat frequency $\left(f D / U_{\infty}=0.2248-0.1508=0.074\right)$ between the cylinder oscillation represented in SPOD modes $(1,2)$ and the natural vortex shedding represented in SPOD modes $(5,6)$.

\subsection{Comparison of Low Order Models for POD and SPOD}

A low order model of the flow field dynamics was constructed from the first four mode pairs for both POD and SPOD decompositions of the wake, as described by Equation 8 . Figure 5 shows the Reynolds stresses from the raw PIV images, and compares the residuals of the two low order models. The Reynolds stress residuals for the two LOMs are similar overall; however, the SPOD-based LOM has slightly higher $u^{\prime} u^{\prime}$ and $v^{\prime} v^{\prime}$ residuals in the near wake (approximately $-1.8<y / D<0.4$ and $x / D<0.4$ ), compared to the POD LOM. Based on the lower energy of the SPOD modes compared to the POD modes, it is expected that the residuals will be slightly higher in the frequency constrained SPOD LOM, for the same number of modes reconstructed. Figure 6 shows the vorticity contours of the two LOM for two vector fields in a representative shedding cycle. The two LOM show remarkably similar representations of the coherent dynamics in the flow. The SPOD LOM thus offers an advantage compared to the POD LOM because each of the four mode pairs in the SPOD LOM is associated with a distinct frequency-centered dynamic. Essentially, SPOD has properly separated these four dynamics and removed the uncorrelated stochastic contributions seen in the POD modes, leaving a deterministic coherent representation of the flow, which is desirable from the perspective of further modeling.

\section{Conclusions}

This study considers the application of SPOD analysis to the wake dynamics of the low-mass-ratio VIV of a circular cylinder in the lower branch, at $U^{*}=7.5$. The effect of the SPOD filter length on the coherent modes 

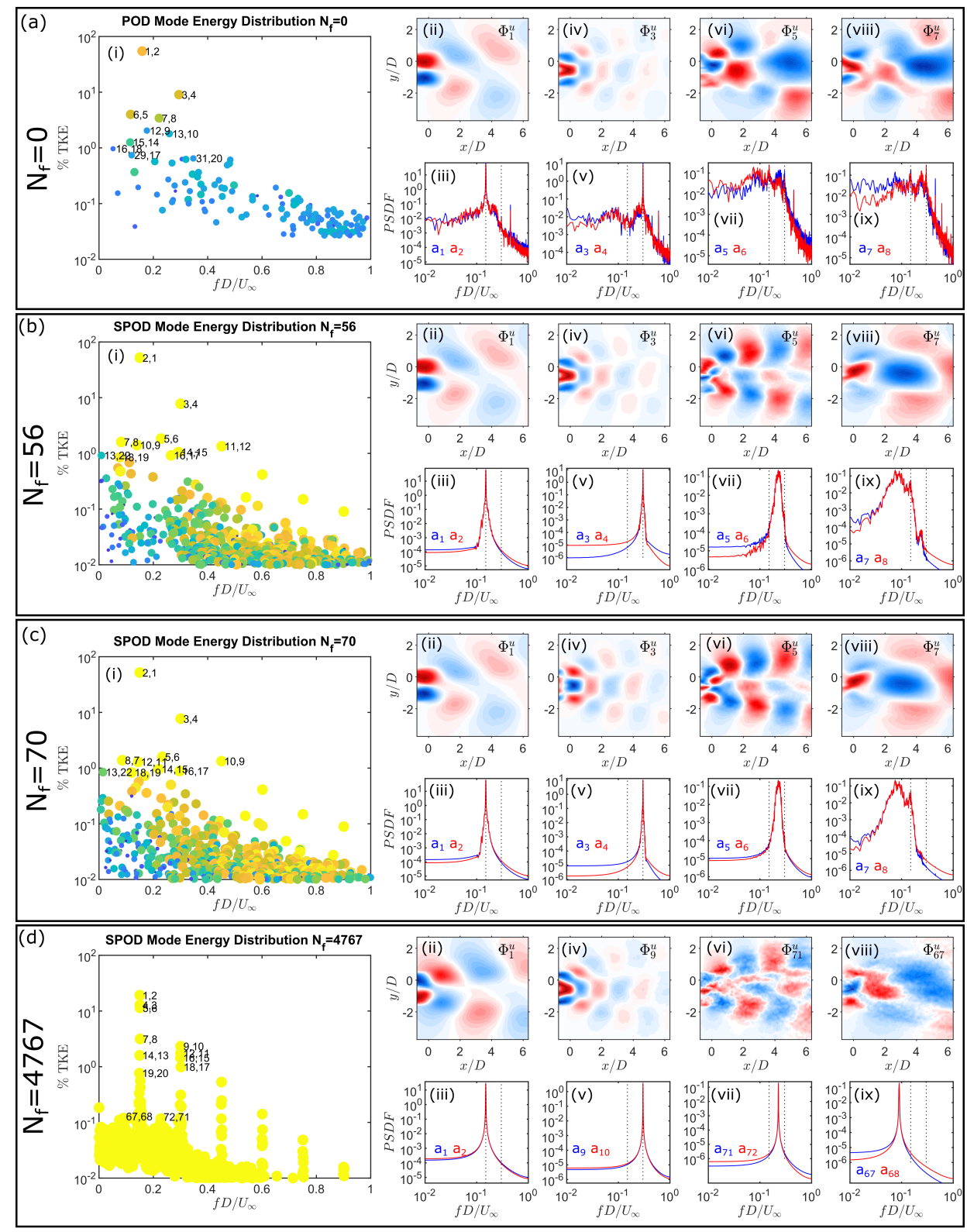

Figure 3: Comparison of SPOD filter lengths $N_{f}=[0,56,70,4767]$ in (a-d). Subplots: (i) Mode pair energy content as a function of the Strouhal number. Circle size and color represent the correlation of the temporal coefficients of the mode pair. (ii,iv,vi,viii) Spatial modes (u-component) for the first 3 most energetic mode pairs from POD, and the same modes in SPOD. (iii,v,vii,ix) Power Spectrum Density Functions (PSDF) of the temporal coefficients for the mode pairs in (ii,iv,vi,viii). The dotted lines at $f D / U_{\infty}=[0.15,0.3]$ correspond to the cylinder oscillation frequency and second harmonic.

was examined, and a filter length of $N_{f}=70$ was chosen to properly separate the four distinct frequencycentered dynamics within the first eight modes, while retaining as much energy as possible. The coherent POD and SPOD modes were compared and the newly separated third SPOD mode pair was found to have a periodicity characteristic of vortex shedding and a peak in the temporal coefficient spectra at $S t=f D / U_{\infty}=$ 0.2248. The fourth SPOD mode pair was found to correspond to the beat frequency between the first and third SPOD mode pairs. While the literature has identified that the wake dynamics within the lower branch are synchronized to the cylinder motion, the present study suggests that some hidden dynamics persist at the Strouhal frequency that were revealed only through application of SPOD analysis. Low order models based 

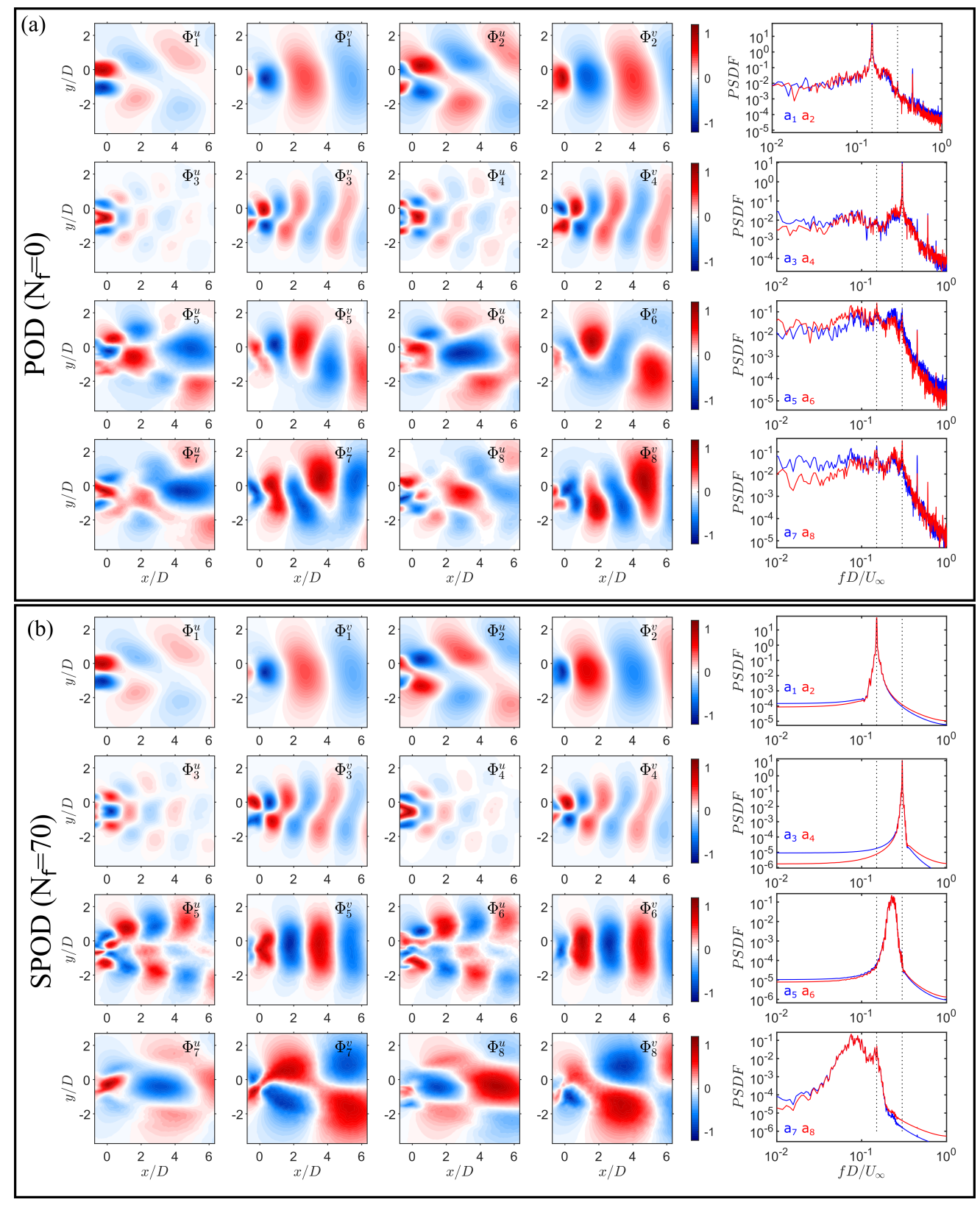

Figure 4: Spatial modes and temporal coefficient spectra for the first three coherent mode pairs identified using POD (a) and SPOD (b). The dashed lines in the spectra are located at $f D / U_{\infty}=0.15,0.30$ and correspond to the cylinder oscillation frequency and second harmonic, respectively.

on the first eight POD and SPOD modes were compared, and it was found that the filtering operation in SPOD removes the uncorrelated stochastic energy contributions seen in the POD modes while producing a comparable representation of the coherent deterministic part of the wake dynamics. Using SPOD to separate the distinct frequency-centered dynamics into unique, interpretable mode pairs will simplify future efforts to develop sparse dynamical models of the flow. 

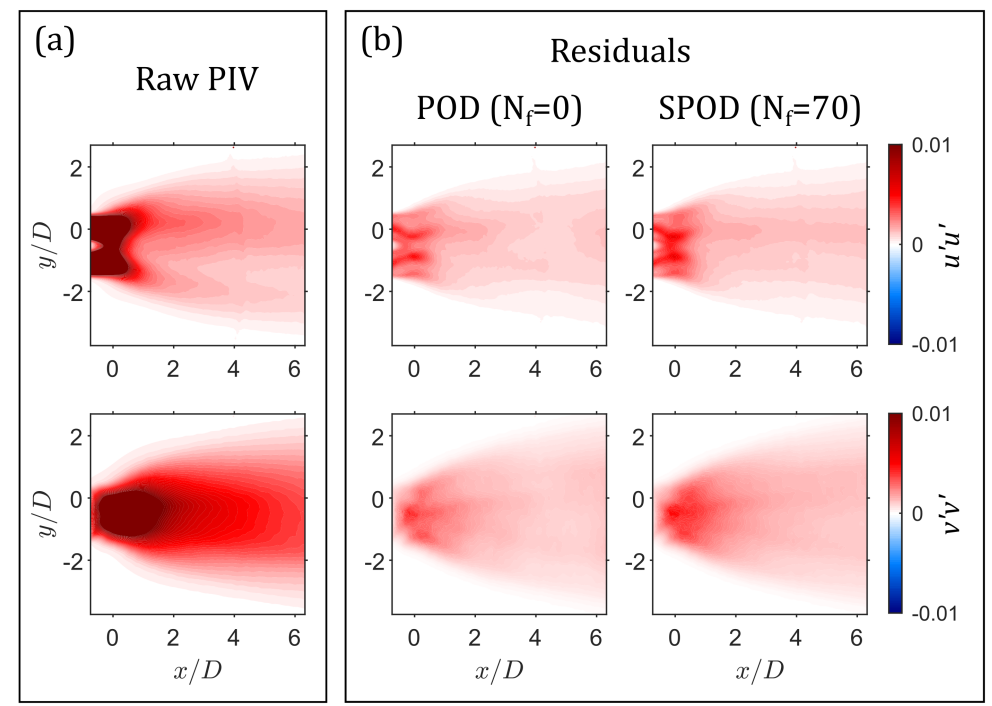

Figure 5: Comparison of Reynolds stresses for the two LOM based on the POD and SPOD decompositions of the wake. Raw data is shown in (a), and the residuals for the POD and SPOD LOM are shown in (b).
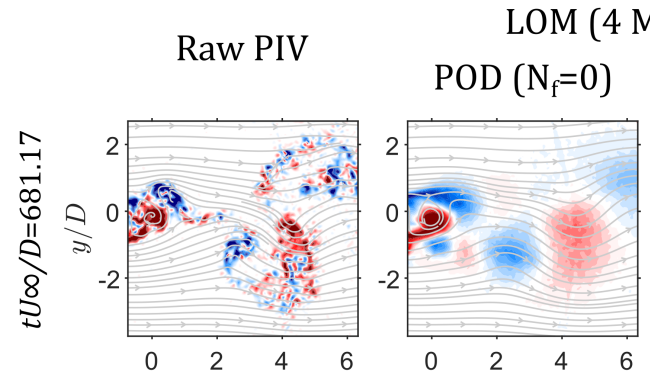

Mode Pairs)
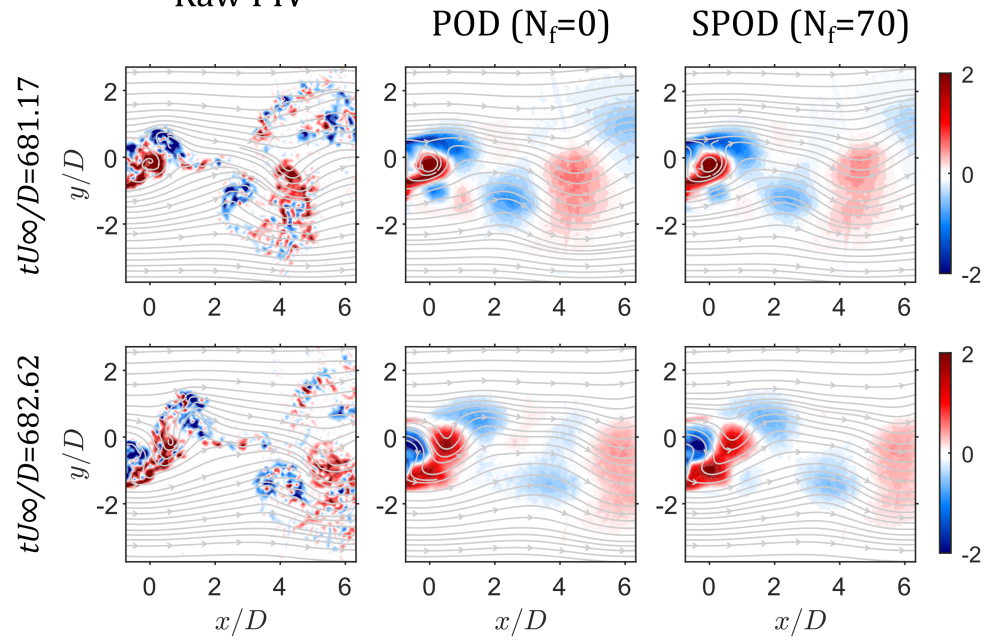

Figure 6: Vorticity contours for the raw PIV velocity fields (left column) and 8-mode low order models (LOM) for POD ( $N_{f}=0$, center column) and SPOD $\left(N_{f}=70\right.$, right column). The contours shown are taken from a representative shedding cycle, for brevity two vector fields are omitted between the shown contours.

\section{References}

Bourgeois JA, Noack BR, and Martinuzzi RJ (2013) Generalized phase average with applications to sensorbased flow estimation of the wall-mounted square cylinder wake. Journal of Fluid Mechanics 736:316350

Derakhshandeh J, Arjomandi M, Cazzolato B, and Dally B (2015) Harnessing hydro-kinetic energy from wake-induced vibration using virtual mass spring damper system. Ocean Engineering 108:115-128

Freire CM, Meneghini JR, Gioria RS, and Assi GRS (2015) Comparison between the koopman modes for the flow around circular cylinder and circular cylinder fitted with helical strakes. in Instability and Control of Massively Separated Flows. pages 117-122 
Govardhan RN and Williamson CHK (2006) Defining the 'modified griffin plot' in vortex-induced vibration: revealing the effect of reynolds number using controlled damping. Journal of Fluid Mechanics 561:147

Hover F, Miller S, and Triantafyllou M (1997) Vortex-induced vibration of marine cables: Experiments using force-feedback. Journal of Fluids and Structures 11:307-326

Khalak A and Williamson C (1997) Fluid forces and dynamics of a hydroelastic structure with very low mass and damping. Journal of Fluids and Structures 11:973-982

Klamo J, Leonard A, and Roshko A (2006) The effects of damping on the amplitude and frequency response of a freely vibrating cylinder in cross-flow. Journal of Fluids and Structures 2:845 - 856. bluff Body Wakes and Vortex-Induced Vibrations (BBVIV-4)

Liberge E and Hamdouni A (2010) Reduced order modelling method via proper orthogonal decomposition (pod) for flow around an oscillating cylinder. Journal of fluids and structures 26:292-311

Mackowski AW and Williamson CH (2011) Developing a cyber-physical fluid dynamics facility for fluidstructure interaction studies. Journal of Fluids and Structures 27:748-757

Morse TL and Williamson CHK (2009) Prediction of vortex-induced vibration response by employing controlled motion. Journal of Fluid Mechanics 634:5

Noack BR, Morzyński M, and Tadmor G, editors (2011) Reduced-Order Modelling for Flow Control. Springer Vienna

Norberg C (2003) Fluctuating lift on a circular cylinder: review and new measurements. Journal of Fluids and Structures 17:57-96

Raissi M, Wang Z, Triantafyllou MS, and Karniadakis GE (2019) Deep learning of vortex-induced vibrations. Journal of Fluid Mechanics 861:119-137

Riches G, Martinuzzi R, and Morton C (2018) Proper orthogonal decomposition analysis of a circular cylinder undergoing vortex-induced vibrations. Physics of Fluids 30:105103

Riches G and Morton C (2018) One degree-of-freedom vortex-induced vibrations at constant reynolds number and mass-damping. Experiments in Fluids 59

Riches GP (2018) Experimental Investigation of Vortex-Induced Vibrations using a Cyber-Physical System. Master's thesis. University of Calgary

Rowley CW (2005) Model reduction for fluids, using balanced proper orthogonal decomposition. International Journal of Bifurcation and Chaos 15:997-1013

Sarpkaya T (2004) A critical review of the intrinsic nature of vortex-induced vibrations. Journal of Fluids and Structures 19:389-447

Sieber M, Paschereit CO, and Oberleithner K (2016) Spectral proper orthogonal decomposition. Journal of Fluid Mechanics 792:798-828

Sirovich L (1987) Turbulence and the dynamics of coherent structures. i. coherent structures. Quarterly of Applied Mathematics 45:561-571

Wieneke B (2015) Piv uncertainty quantification from correlation statistics. Measurement Science and Technology 26:074002

Williamson C and Govardhan R (2004) Vortex-induced vibrations. Annual Review of Fluid Mechanics $36: 413-455$ 